\title{
Processing of Abstract Ordinal Knowledge in the Horizontal Segment of the Intraparietal Sulcus
}

\author{
Wim Fias, ${ }^{1,2}$ Jan Lammertyn, ${ }^{1}$ Bernie Caessens, ${ }^{1}$ and Guy A. Orban ${ }^{3}$ \\ ${ }^{1}$ Department of Experimental Psychology and ${ }^{2}$ Ghent Institute for Functional and Metabolic Imaging, Ghent University, 9000 Gent, Belgium, and \\ ${ }^{3}$ Laboratorium voor Neuro- en Psychofysiologie, Medical School, Katholieke Universiteit Leuven, 3000 Leuven, Belgium
}

The anterior intraparietal sulcus, and more specifically its horizontal segment (hIPS), is known to play a crucial role in the cognitive representation of numerical quantity. Whether the involvement of hIPS is restricted to the processing of numerical information or generalizes to non-numerical ordinal dimensions remains an open question. Using functional magnetic resonance imaging during comparison tasks, we demonstrate that the hIPS is equally responsive to numbers and letters, indicating that hIPS is also involved in the representation and processing of non-numerical ordinal series. This extends the numerical processing function of IPS into the realm of abstract knowledge processing.

Key words: fMRI; parietal cortex; anterior intraparietal sulcus; ordinality; number processing; abstract cognition

\section{Introduction}

The human capacity to understand numerical symbols is thought to have evolved from basic neural systems that endow various species with a number sense. In humans, different parietal regions contribute to numerical processing tasks, but only the anterior part of the horizontal segment of the intraparietal sulcus (hIPS) codes numerical quantity, thereby generating a basic sense of number (Dehaene et al., 2003). Indeed, lesions and atrophies involving hIPS impair normal number sense (Dehaene and $\mathrm{Pi}$ azza, 2003). Furthermore, hIPS activation in functional magnetic resonance imaging (fMRI) is modulated by the numerical distance between numbers during number comparison tasks (Pinel et al., 2001) and even during passive viewing (Piazza et al., 2004, 2007). This distance-related activation is explained in detail by the properties of numerosity-selective neurons recorded in the putative monkey homolog of hIPS (Nieder and Miller, 2004).

Numerical knowledge, however, requires not only a representation of quantity but also the ordinal relationship between quantities. Hence, the specific role of hIPS in number processing might be to represent ordinality rather than just quantity (Nieder, 2005). Previous studies found systematically stronger hIPS activation during the processing of numbers than for nonnumerical ordinal dimensions such as letters (Eger et al., 2003), animal ferocity (Thioux et al., 2005), or body part position (Le Clec'H et al., 2000), suggesting that hIPS activation does not relate to the processing of ordinal information in general, but of quantitative information specifically (Piazza and Dehaene, 2004). These studies, however, cannot be considered conclusive.

Received May 7, 2007; revised June 21, 2007; accepted July 5, 2007.

This work was supported by Grant P6/29 from the Interuniversity Attraction Poles program of the Belgian federal government.

Correspondence should be addressed to Wim Fias, Department of Experimental Psychology, Ghent University, H. Dunantlaan 2,9000 Gent, Belgium. E-mail: wim.fias@ugent.be.

D01:10.1523/JNEUROSCI.2076-07.2007

Copyright $\odot 2007$ Society for Neuroscience $\quad$ 0270-6474/07/278952-05\$15.00/0
Body position or animal ferocity may not be stored as ordinal dimensions in long-term memory, but may be created only for temporary task demands. Letters were investigated using an order-irrelevant letter identification task, and explicit processing of order may be required to activate hIPS.

Inspired by the close behavioral similarities between the processing of numerical and non-numerical series like letters, months, and days of the week (Gevers et al., 2003, 2004), we asked participants to compare two letters in terms of their position in the alphabet and compared the blood oxygenation leveldependent (BOLD) activations to those obtained during the comparison of two-digit numbers. Each comparison task was controlled by a dimming detection task using identical sensory input and motor responses. Because alphabetic order is stored in long-term memory and because the comparison task requires explicit processing of order, letter comparison is better suited for demonstrating the hIPS involvement in the processing of nonnumerical sequences than previous attempts. To separate nonspecific neural resources required simply for the comparison of two stimuli, we included a color saturation comparison task (which of two color stimuli is most saturated?), because color and luminance comparisons do not engage hIPS (Pinel et al., 2004; Shuman and Kanwisher, 2004).

Predictions are straightforward. If hIPS is selective for the number category, its activation should be modulated by a stimulus (number vs letter) by task (comparison vs dimming) interaction. If, however, the function of hIPS extends to the processing of letters, then hIPS activation should be detected in a conjunction analysis between letter and number comparison. Before accepting either of these two possibilities, an additional requirement is that the hIPS region is not involved in saturation comparison. This will be verified with task $\times$ stimulus interaction analyses involving saturation and either numbers or letters. Moreover, a three-way conjunction analysis between numbers, 
letters, and saturation will be performed to detect cortical regions that provide general resources to the comparison task.

\section{Materials and Methods}

Subjects. Seventeen volunteers ( 9 female, 13 right-handed; age, 20-37 years) participated in the experiment. All gave written informed consent as approved by the ethics committee of the Medical Department of Ghent University. None of the subjects had a history of neurological or psychiatric illness.

Stimuli and materials. Stimulus presentation and recording of behavioral data were controlled by a $\mathrm{C}$ program using the Tscope library (Stevens et al., 2006), running on a Pentium 4 laptop. Stimuli were projected onto a translucent screen placed at the back of the magnet bore using a Sony (Tokyo, Japan) VPL-PX20 liquid crystal display projector. Participants viewed the screen through a mirror mounted on the head coil, at a distance of $\sim 30 \mathrm{~cm}$ from the eyes.

Three types of stimuli were used: numbers, letters, and colored squares. In each trial, two stimuli of the same type were presented on either side of a fixation cross $\left(0.38 \times 0.38^{\circ}\right)$ at an eccentricity of $10^{\circ}$. Numbers and letters were presented in white. The height of letters and numbers was set to $1.02^{\circ}$. The widths of the numbers varied from 2.2 to $1.24^{\circ}$, and that of the letters varied from 0.19 to $1.05^{\circ}$. Saturation squares measured $2.58 \times 2.58^{\circ}$. To compensate for brightness differences in the saturation condition, the background color was varied on a trial-by-trial basis between light gray [all red-green-blue (RGB) values 166] and dark gray (all RGB values 80 ).

We used two tasks: comparison and dimming detection. Combined with the different stimulus types, this resulted in six possible conditions: comparing numbers, letters, or saturation levels and detecting the dimmed number, letter, or square.

To form the stimulus pairs for the comparison tasks, the first item was randomly chosen from a predefined set, either 24 letters (B to Y), 89 numbers (10-98), or any combination of hue and saturation in the huesaturation-brightness (HSB) color space with a constant brightness value of $96 \%$ in HSB space. To present the selected colors on the computer screen, they were converted from HSB space to the RGB color model. Once the first item was defined, the second item of the pair was chosen so as to differ by a distance that yielded $80 \%$ performance accuracy, as determined during a training session preceding the scanning. Averaged over subjects, the numerical distance was 9.75 (SD, 2.60), the alphabetical distance was 10.21 (SD, 0.80 ) positions in the alphabet, and saturation distance was a value of $10 \%$ in HSB space (SD, 3.00). Stimulus selection was identical in the corresponding dimming tasks.

The brightness of one of the two stimuli was reduced for a period of 75 ms. The amplitude of this luminance decrease was adjusted for each participant during the preliminary practice session to obtain $80 \%$ detection accuracy in the dimming tasks. Averaged over participants, values of 29.44 (SD, 4.51), 29.65 (SD, 4.45), and 11.87 (SD, 2.92) percentage in HSB space were subtracted from the initial value of $96 \%$ in HSB space for number, letter, and saturation stimuli, respectively. Dimming occurred randomly on either side. Dimming also occurred in the comparison trials.

Procedure. During two $1 \mathrm{~h}$ training sessions (one the day before and one immediately before scanning), participants were trained until they reached a stable performance level of $\sim 80 \%$ accuracy for each condition. This was done on an individual basis by adapting the amplitude of dimming and the distance between numbers, letters, and saturation levels. In this way, we tried to avoid differences in task difficulty that might covary with our predictions.

The actual experiment was organized into five runs, each consisting of 12 blocks (with all of the six conditions appearing twice) of 16 trials. Feedback was given between runs. The order of the blocks in a run was randomized with the restriction that the same condition could not occur in two successive blocks.

Each block began with a fixation period of $5.6 \mathrm{~s}$. Immediately thereafter, a summary of the instructions for that block was presented for $2.8 \mathrm{~s}$, followed by a series of 16 trials lasting $1350 \mathrm{~ms}$ each.

All trials started with fixation screen of $250 \mathrm{~ms}$. Next, a stimulus pair was presented for $250 \mathrm{~ms}$. During stimulus presentation, dimming was applied to one of the two stimuli. Dimming onset occurred between 100 and $150 \mathrm{~ms}$ after stimulus onset and lasted for $75 \mathrm{~ms}$. After stimulus presentation, the screen was replaced by a fixation-cross screen for 800 $\mathrm{ms}$ and was followed by an intertrial interval of $50 \mathrm{~ms}$.

The task of the participants during comparison blocks was to decide which of two numbers was the larger, which of two letters came later in the alphabet, or which square was most saturated. In the dimming task, participants had to respond to the stimulus that dimmed. For all conditions, responses were made by pressing a response key with the hand corresponding to the side of the target stimulus. Responding was possible from the onset of stimulus presentation until 1050 ms thereafter. This short response schedule was chosen to prevent participants from using complex strategies such as numerical labeling of the letters to solve the task. A pilot study showed that, after having been trained to $80 \%$ correct in the letter comparison task, participants $(n=3)$ needed an average of $2276 \mathrm{~ms}$ to orally produce the number that corresponded to a letter's position in the alphabet (targets $\mathrm{B}$ to $\mathrm{Y}$ ), with the average responses deviating 1.65 from the correct answer.

fMRI data acquisition. Imaging was performed on a Siemens (Munich, Germany) 3T Trio scanner using a standard eight-channel head coil. After automatic shimming of the magnetic field, a three-dimensional (3D) high-resolution T1 anatomical image was acquired for coregistration with the functional images [3D magnetization-prepared rapidacquisition gradient echo, 176 slices; slice thickness, $0.90 \mathrm{~mm}$; in-plane resolution, $0.9 \times 0.9 \mathrm{~mm}$; repetition time (TR), $1550 \mathrm{~ms}$; echo time $(\mathrm{TE})$, $2.89 \mathrm{~ms}]$. Next, functional volumes were acquired using a multiple slice $\mathrm{T} 2^{*}$-weighted echo planar imaging (EPI) sequence with the following parameters: TR, $2800 \mathrm{~ms}$; TE, $33 \mathrm{~ms}$; flip angle, $90^{\circ}$; in-plane resolution, $3 \times 3 \mathrm{~mm}$; matrix dimensions, $64 \times 64$, resulting in a field of view of $192 \times 192 \mathrm{~mm}$. Slice thickness was $2 \mathrm{~mm}$, with an interslice gap of 50\%. Forty slices covered the entire brain. Each of the five runs produced 132 volumes.

Statistical analysis of fMRI data. Data processing and analysis were performed using SPM2 (http://www.fil.ion.ucl.ac.uk/spm/software/ spm2/). All images were coregistered with the participant's corresponding anatomical (T1-weighted) images and realigned to the first volume. The resulting images were normalized with a 12-parameter affine transformation into SPM2's Montreal Neurological Institute EPI $3 \times 3 \times 3$ $\mathrm{mm}$ template using the corresponding anatomical image as a reference, smoothed using a $6 \mathrm{~mm}$ full-width at half-maximum Gaussian kernel, and high-pass filtered with a frequency cutoff at $369.6 \mathrm{~s}$. The BOLD response in each block was modeled in a general linear model with a separate regressor for each task condition and one for the instructions. Predictors were convolved with a canonical hemodynamic response function to form covariates in a general linear model.

Whole-brain group analyses were performed according to the random effects procedure, using the single-subject contrast images as input. Group statistical parametric maps (SPMs) were generated using a onesample $t$ test with a voxel threshold of $p<0.005$. Conjunction analyses were performed using the minimum statistic compared with the conjunction null method as proposed by Nichols et al. (2005). Whole-brain interaction analyses between task (comparison vs dimming) and stimulus type were inclusively masked with the main effect of task (comparison $>$ dimming at a low threshold of $p<0.05$ and with an extent threshold of five voxels) to exclude voxels that contribute to dimming more than to comparison. For region of interest (ROI) analyses, ROIs were defined by the random effect brain activations, obtained from the conjunction between number and letter comparison, compared with their respective dimming controls $(p<0.005) . \beta$ values of these ROIs were determined using Marsbar 0.38 (Brett et al., 2002) and analyzed with ANOVA $(p<0.05)$ to investigate interactions with the saturation tasks. SPMs were mapped to the human population-average, landmark and surface-based (PALS) atlas using the average voxel algorithm (neighbor box size, $10 \times 10 \mathrm{~mm}$ ) via Caret (Van Essen et al., 2001; http://brainmap.wustl.edu/caret). 


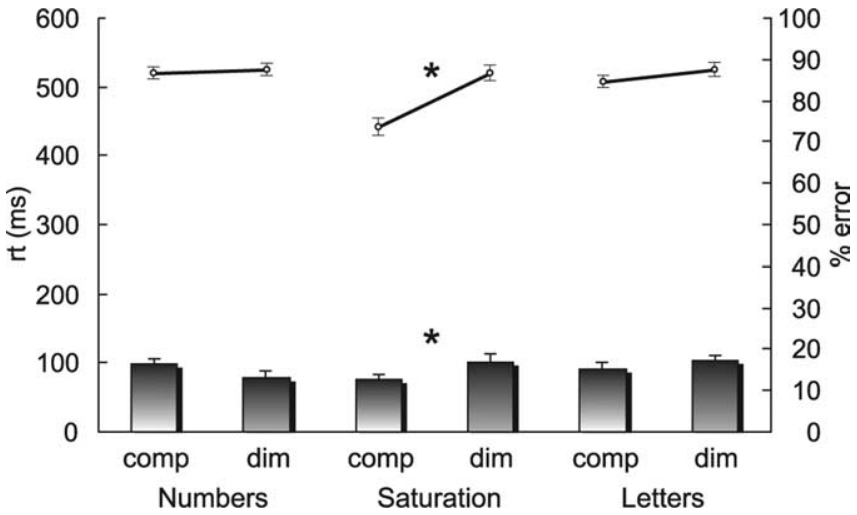

Figure 1. Behavioral results. Shown are reaction times (lines) and percentage error (bars) \pm SEM. Differences were analyzed using dependent-sample $t$ tests ( $\left.{ }^{*} p<0.05\right)$. comp, Comparison task; dim, dimming task.

\section{Results}

\section{Behavioral}

Reaction times (RTs) and accuracy were analyzed with a 2 (task: comparison and dimming) $\times 3$ (stimulus type: numbers, letters, and saturation) repeated-measures ANOVA. For RTs, the main effects of task $\left[F_{(1,16)}=16.75\right.$; mean squared error $(\mathrm{MSE})=1660$; $p<0.005]$, stimulus type $\left(F_{(2,32)}=40.91 ; \mathrm{MSE}=433 ; p<\right.$ $0.0005)$ as well as the interaction between task and stimulus type $\left(F_{(2,32)}=31.44 ; \mathrm{MSE}=393 ; p<0.005\right)$ were significant. Planned comparisons for all stimulus types between the two tasks revealed that the difference between the comparison and the dimming control task was only significant for saturation $\left(F_{(1,16)}=47.48\right.$; MSE $=1046.88 ; p<0.0005$ ) (Fig. 1).

In the analysis of the accuracy data, only the task $\times$ stimulus type interaction reached significance $\left(F_{(2,32)}=4.89\right.$; MSE $=$ $27.88 ; p<0.05)$. Planned comparisons indicated that the difference between the comparison and the dimming control task was significant for saturation $\left(F_{(1,16)}=4.69 ; \mathrm{MSE}=36.2 ; p<0.05\right)$, tended toward significance for numbers $\left(F_{(1,16)}=4.09\right.$; $\mathrm{MSE}=$ 23.37; $p<0.07)$, and was not significant for letters.

\section{Imaging}

Number and letter comparison engaged highly similar neural networks. Whole-brain interaction analyses that looked for areas in which the number comparison versus number dimming contrast is stronger than the letter comparison versus letter dimming contrast (or vice versa) revealed no number- or letter-specific activations apart from enhanced activity of visual areas (calcarine sulcus, $6,-72$, -6 ; left inferior occipital gyrus, $-39,-87,-6$; left fusiform gyrus, $-24,-78,-9$; Talairach coordinates) in the number comparison task, probably because of the higher visual complexity of two-digit numbers relative to individual letters.

A conjunction analysis of the comparison versus dimming contrast for the number and letter conditions revealed a common network of occipital, temporal, parietal, and frontal regions (Fig. 2 ). Within parietal cortex, an anterior and a posterior region of the IPS in both hemispheres could be distinguished. Additional analyses revealed distinct functional profiles for the anterior and posterior IPS clusters.

The left and right anterior clusters coincide with the putative human homolog of the anterior intraparietal area (Grefkes et al., 2002) and overlap with the bilateral barycenters of hIPS activation in number processing tasks as determined in a recent metaanalysis (Dehaene et al., 2003) (Fig. 2). These anterior IPS clusters were further inspected by ROI analyses. A $2 \times 2$ ANOVA with the factors stimulus type (numbers vs saturation) and task (comparison vs dimming) showed a significant interaction in the left hemisphere ROI $\left(F_{(1,16)}=4.47\right.$; MSE $\left.=0.0211 ; p=0.05\right)$ and in the right hemisphere $\operatorname{ROI}\left(F_{(1,16)}=4.71 ; \mathrm{MSE}=0.0215 ; p<\right.$ $0.05)$. The same type of ANOVA, but with saturation and letters as the stimulus type conditions, revealed a significant interaction in the left hemisphere ROI $\left(F_{(1,16)}=14.64\right.$; MSE $=0.0102 ; p<$ 0.005 ) (Fig. $3 a$ ) and a trend toward significance in the right hemisphere $\left(F_{(1,16)}=3.77 ;\right.$ MSE $\left.=0.01743 ; p<0.07\right)($ Fig. $3 b)$. These interactions indicate that the hIPS region is not involved in saturation comparison.

The fact that behavioral performance was faster and more accurate in saturation comparison as opposed to saturation dimming (Fig. 1) does not weaken this conclusion. The behavioral difference cannot be taken as evidence that general task difficulty drives the BOLD differences in hIPS, because in that case there should be a stronger neural response in saturation dimming than in saturation comparison. This is clearly not the case. Nevertheless, one could argue that the absence of enhanced hIPS activation in saturation comparison can be attributed to the ease with which a specific component of the comparison task is performed. This alternative explanation is untenable in light of an ANCOVA analysis comparing activation in hIPS during the saturation comparison and dimming tasks, using the reaction time difference between saturation comparison and dimming as a covariate: in neither the left nor the right hIPS ROIs did the covariate interact with the task factor (left hIPS, $F_{(1,15)}=0.641 ; p=0.44$; right hIPS, $\left.F_{(1,15)}=0.15 ; p=0.70\right)$.

Whereas the anterior IPS cluster is thus specifically related to number and letter comparison, the posterior IPS cluster shows a different functional profile. The fact that this posterior IPS region was involved in the three-way conjunction for saturation, number, and letter stimuli (Fig. 2) indicates that this region has a more general function, perhaps related to response selection (Jiang and Kanwisher, 2003). It is noteworthy that precentral cortex also exhibits a dissociation between order-related processing (bilateral dorsal parts) and general-purpose processing (left ventral part).

\section{Discussion}

The present experiment was designed to test the hypothesis that the involvement of the hIPS in number processing reflects neural processing that is selective for the number category. The results clearly show that the comparisons of letters and of numbers are subserved by a highly similar neural network comprising occipital cortex, temporal cortex, intraparietal sulci, and frontal areas. Whereas a large part of this network serves general-purpose processing (and was thus also recruited by saturation comparison), another part of this network is dedicated to number and letter processing. Most importantly, this specific network comprises the anterior part of the hIPS, which has been identified as the crucial brain area supporting number sense (Dehaene et al., 2003). The involvement of the hIPS in the processing of nonnumerical ordinal series, like the alphabet, is in disagreement with the hypothesis that hIPS is selectively engaged in the processing of numerical information and argues in favor of a common neural mechanism. Of course, the joint activation of hIPS as detected with fMRI does not imply that the same neurons are involved in processing of numerical and non-numerical order. Hence, (partial) neural segregation may still exist within this network. The precision with which neurons in IPS are tuned to numerical magnitude (Nieder et al., 2004) suggests a high degree 


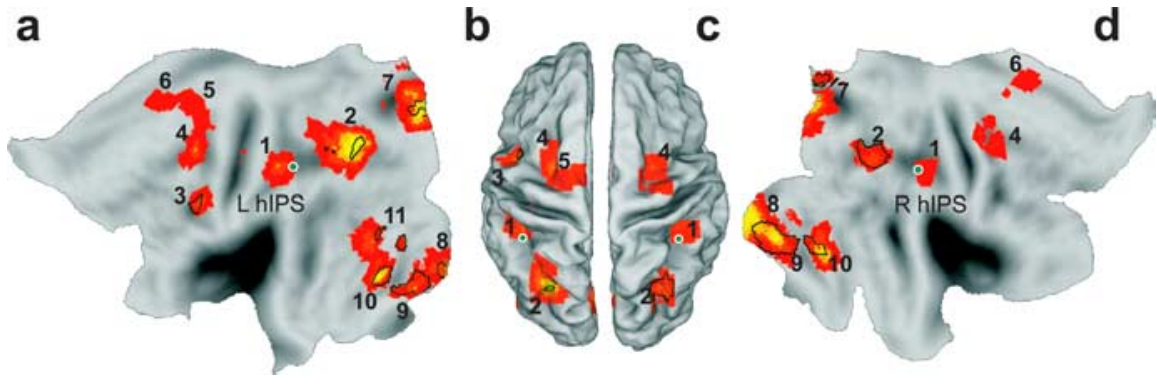

Figure 2. Imaging results. $\boldsymbol{a}-\boldsymbol{d}$, Statistical parametric map of conjunction of numbers and letters $(p<0.005)$ mapped on a dorsal view $(\boldsymbol{b}, \boldsymbol{c})$ and flat map $(\boldsymbol{a}, \boldsymbol{d})$ of the human PALS atlas (see Materials and Methods). Black contours indicate the result of the conjunction of numbers, letters, and saturation $(p<0.005)$. Green circles indicate barycenters of the Dehaene et al. (2003) meta-analysis. 1, hIPS (-39, -39, 36 and 45, -36, 48); 2, posterior parietal (-27, $-69,39$ and 30, $-69,42)$; 3, ventral precentral $(-42,3,30) ; 4$, dorsal precentral $(-24,-3,57$ and $27,0,51) ; 5$, superior frontal $(-18,-9,72) ; 6$, supplementary motor area $(-6,3,53$ and $12,3,45) ; 7$, dorsal calcarine $(-3,-75,10$ and $10,-65,13) ; 8$, ventral calcarine $(-7,-72,5$ and $11,-68,11) ; 9$, inferior temporal $(-14,-48,-8$ and $9,-56,-1) ; 10$, fusiform $(-24,-50,-16$ and $29,-47,-17) ; 11$, inferior temporal $(-44,-68,-13)$. Coordinates are in Talairach space. a

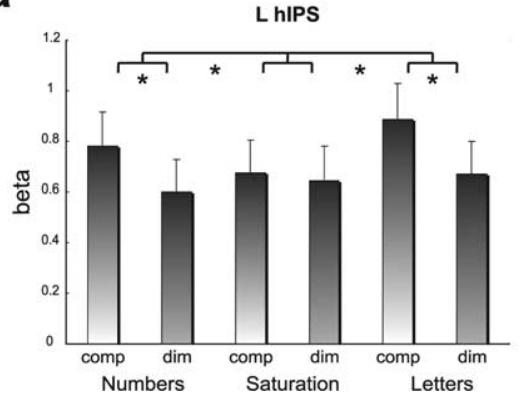

b

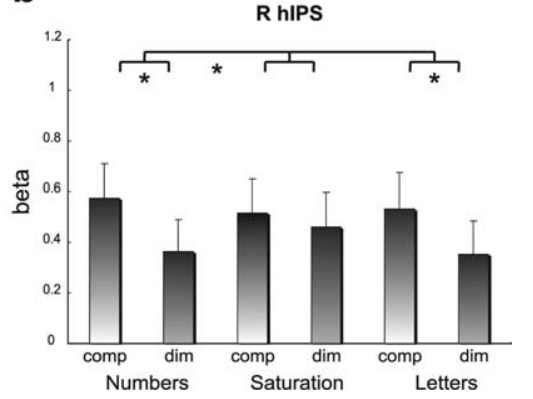

Figure 3. hIPS ROI profiles. $\boldsymbol{a}, \boldsymbol{b}$, Profiles show average $\beta$ values compared with fixation baseline for left (L) hIPS (a) and right (R) hIPS $(\boldsymbol{b})$ ROls defined by the conjunction of numbers and letters, thresholded at $p<0.005$. Task $\times$ stimulus type interactions and task differences for each type of stimulus were analyzed using ANOVA and dependent-samples $t$ tests, respectively $\left({ }^{*} p<\right.$ 0.05). comp, Comparison task; dim, dimming task.

of functional specialization and makes it unlikely that the same neurons also encode non-numerical order. Further support for partial segregation comes from neuropsychological studies with patients suffering from Gerstmann syndrome after parietal lesions. Although a joint impairment of the processing of numerical and non-numerical order has been described (Cipolotti et al., 1991), a case with preserved number comparison in the presence of impaired letter comparison has also been documented (Turconi and Seron, 2002).

The demonstration of hIPS involvement in alphabetical order processing extends the functional role of the IPS into the realm of abstract cognition. The level of abstraction of alphabetical order goes well beyond the levels of abstraction that have so far been established with number processing. In monkeys, Nieder et al. (2006) described IPS neurons that coded number, independent of the temporal or spatial presentation of quantity. In humans, hIPS has been shown to code numerical magnitude independent of the stimulus type that conveys the magnitude information (Fias et al., 2003; Cohen Kadosh et al., 2007). Interestingly, a recent fMRI study found crossnotational adaptation of the BOLD signal between dot patterns and digits (Piazza et al., 2007). Such cross-notational effects are in line with a recent computational model proposing how symbolic number comprehension arises from associating symbols with sensorybased, number-selective neurons (Verguts and Fias, 2004). Hence, although a considerable level of abstraction is realized by the hIPS, its involvement in symbolic number processing is still closely linked to sensory-based neural mechanisms in the sense that symbolic num-

\section{References}

ber comprehension is tightly grounded in the general ability to represent visual numerosity. For alphabetical position, however, there is no equivalent in the sensory experience of the physical world. Rather, it is a dimension for which the ordinal organization is largely determined by abstract principles, possibly dependent on linguistic processing (as in reciting the alphabet). The fact that letter comparison engages the same neural network as number comparison suggests that the brain accomplishes the processing of information at high levels of abstraction by recruiting brain areas that have ontogenetically or phylogenetically evolved to process information at lower levels of abstraction that are ultimately grounded in sensory experience.

Undoubtedly, the capacity to code abstract information is limited by anatomical and physiological constraints. As such, the neural substrate for performing polymodal processing in anterior intraparietal areas (Bremmer et al., 2001; Grefkes et al., 2002) may be the predecessor of higher levels of abstraction. The neural computations performed by neurons in polymodal areas serve coordinate transformations. Single-cell studies indicate that although many neurons in polymodal areas respond to different sensory modalities, there is generally a dominant sensory modality [e.g., visual over auditory in the lateral intraparietal area (Stricanne et al., 1996); somatosensory over visual in the ventral intraparietal area (Avillac et al., 2005)]. Neural processing in these polymodal areas is consistent with coordinate transformations converting the coordinates of the nondominant sense into the coordinates of the dominant sense of that area (Pouget et al., 2002; Avillac et al., 2005). Considering the fact that ordinal cognitive dimensions are essentially unidimensional coordinate systems, our findings can be accounted for by the general principles of polymodal neural processing as described above. It is plausible to assume that the number coordinate system is dominant because of its grounding in sensory processing (Verguts and Fias, 2004), whereas non-numerical coordinate systems are subordinate (in the case of abstract ordinal dimensions, such as the alphabet, because of the absence of direct sensory associations) and are transformed to the numerical coordinate system.

Although still speculative, such a mechanism can provide a biologically plausible account for our observation that the processing of a non-numerical dimension as abstract as the alphabet converges with the more concrete numerical dimension in the anterior part of the IPS. The anatomical observation that these anterior structures of IPS are greatly expanded in humans (reaching high levels of abstraction) compared with monkeys (less endowed with the ability to abstract) (Orban et al., 2006) supports this view.

Avillac M, Deneve S, Olivier E, Pouget A, Duhamel JR (2005) Reference frames for representing visual and tactile locations in parietal cortex. Nat Neurosci 8:941-949. 
Bremmer F, Schlack A, Shah NJ, Zafiris O, Kubischik M, Hoffmann KP, Zilles K, Fink GR (2001) Polymodal motion processing in posterior parietal and premotor cortex: a human fMRI study strongly implies equivalencies between humans and monkeys. Neuron 29:287-296.

Brett M, Anton JL, Valabregue R, Poline JB (2002) Region of interest analysis using an SPM toolbox. Paper presented at the 8th International Conference on Functional Mapping of the Human Brain, Sendai, Japan, June.

Cipolotti L, Butterworth B, Denes G (1991) A specific deficit for numbers in a case of dense acalculia. Brain 114:2619-2637.

Cohen Kadosh R, Cohen Kadosh K, Kaas A, Henik A, Goebel R (2007) Notation-dependent and -independent representations of numbers in the parietal lobes. Neuron 53:87-94.

Dehaene S, Piazza M, Pinel P, Cohen L (2003) Three parietal circuits for number processing. Cogn Neuropsychol 20:487-506.

Eger E, Sterzer P, Russ MO, Giraud AL, Kleinschmidt A (2003) A supramodal number representation in human intraparietal cortex. Neuron 37:719-725.

Fias W, Lammertyn J, Reynvoet B, Dupont P, Orban GA (2003) Parietal representation of symbolic and nonsymbolic magnitude. J Cogn Neurosci 15:47-56.

Gevers W, Reynvoet B, Fias W (2003) The mental representation of ordinal sequences is spatially organized. Cognition 87:B87-B95.

Gevers W, Reynvoet B, Fias W (2004) The mental representation of ordinal sequences is spatially organized: evidence from days of the week. Cortex 40:171-172.

Grefkes C, Weiss PH, Zilles K, Fink GR (2002) Crossmodal processing of object features in human anterior intraparietal cortex: an fMRI study implies equivalencies between humans and monkeys. Neuron 35:173-184.

Jiang YH, Kanwisher N (2003) Common neural substrates for response selection across modalities and mapping paradigms. J Cogn Neurosci 15:1080-1094.

Le Clec'H G, Dehaene S, Cohen L, Mehler J, Dupoux E, Poline JB, Lehericy S, van de Moortele PF, Le Bihan D (2000) Distinct cortical areas for names of numbers and body parts independent of language and input modality. NeuroImage 12:381-391.

Nichols T, Brett M, Andersson J, Wager T, Poline JB (2005) Valid conjunction inference with the minimum statistic. NeuroImage 25:653-660.

Nieder A (2005) Counting on neurons: the neurobiology of numerical competence. Nat Rev Neurosci 6:1-14.

Nieder A, Miller EK (2004) A parieto-frontal network for visual numerical information in the monkey. Proc Natl Acad Sci USA 101:7457-7462.
Nieder A, Diester I, Tudusciuc O (2006) Temporal and spatial enumeration processes in the primate parietal cortex. Science 313:1431-1435.

Orban GA, Claeys K, Nelissen K, Smans R, Sunaert S, Todd JT, Wardak C, Durand JB, Vanduffel W (2006) Mapping the parietal cortex of human and non-human primates. Neuropsychologia 44:2647-2667.

Piazza M, Dehaene S (2004) From number neurons to mental arithmetic: the cognitive neuroscience of number sense. In: The cognitive neurosciences, Ed 3 (Gazzaniga MS, ed), pp 865-875. Cambridge, MA: MIT.

Piazza M, Izard V, Pinel P, Le Bihan D, Dehaene S (2004) Tuning curves for approximate numerosity in the human intraparietal sulcus. Neuron 44:547-555.

Piazza M, Pinel P, Le Bihan D, Dehaene S (2007) A magnitude code common to numerosities and number symbols in human intraparietal cortex. Neuron 53:293-305.

Pinel P, Dehaene S, Riviere D, LeBihan D (2001) Modulation of parietal activation by semantic distance in a number comparison task. NeuroImage 14:1013-1026.

Pinel P, Piazza M, Le Bihan D, Dehaene S (2004) Distributed and overlapping cerebral representations of number, size, and luminance during comparative judgments. Neuron 41:983-993.

Pouget A, Deneve S, Duhamel JR (2002) A computational perspective on the neural basis of multisensory spatial representations. Nat Rev Neurosci 3:741-747.

Shuman M, Kanwisher N (2004) Numerical magnitude in the human parietal lobe: tests of representational generality and domain specificity. Neuron 44:557-569.

Stevens M, Lammertyn J, Verbruggen F, Vandierendonck A (2006) Tscope: a C library for programming cognitive experiments on the MS Windows platform. Behav Res Methods 38:280-286.

Stricanne B, Andersen RA, Mazzoni P (1996) Eye-centered, head-centered, and intermediate coding of remembered sound locations in area LIP. J Neurophysiol 76:2071-2076.

Thioux M, Pesenti M, Costes N, De Volder A, Seron X (2005) Taskindependent semantic activation for numbers and animals. Brain Res Cogn Brain Res 24:284-290.

Turconi E, Seron X (2002) Dissociation between order and quantity meanings in a patient with Gerstmann syndrome. Cortex 38:911-914.

Van Essen DC, Drury HA, Dickson J, Harwell J, Hanlon D, Anderson CH (2001) An integrated software suite for surface-based analyses of cerebral cortex. J Am Med Inform Assoc 8:443-459.

Verguts T, Fias W (2004) Representation of number in animals and humans: a neural model. J Cogn Neurosci 16:1493-1504. 